Received Date : 28-Sep-2016

Revised Date : 08-Nov-2016

Accepted Date : 24-Nov-2016

Article type :Original Papers

\title{
A comprehensive assessment of cognitive function in the common genetic generalised epilepsy syndromes
}

Loughman, A. ${ }^{\mathrm{a}, \mathrm{b} *}\left(\mathrm{BSc}\left[\right.\right.$ Hons]/BA), Bowden, S.C. ${ }^{\mathrm{a}, \mathrm{c}}(\mathrm{PhD})$, D’Souza, W.J. ${ }^{\mathrm{d}}(\mathrm{PhD})$

${ }^{a}$ Melbourne School of Psychological Sciences, University of Melbourne, Parkville, VIC 3010, Australia.

${ }^{b}$ School of Health and Biomedical Sciences, RMIT University, PO Box 71, Bundoora, VIC 3083, Australia.

cDepartment of Clinical Neurosciences, St Vincent's Hospital Melbourne, 41 Victoria

Parade, Fitzroy VIC 3065, Australia.

${ }^{d}$ Department of Medicine, St Vincent's Hospital, The University of Melbourne, 41 Victoria

Parade, Fitzroy VIC 3065, Australia.

*Correspondence: Amy Loughman amy.loughman@rmit.edu.au Fax: + 61393476618

Running title: Cognitive function in GGE

Keywords: epilepsy, genetic generalised epilepsy, cognition, childhood absence epilepsy

Disclosure: AL received an Australian NHMRC Public Health Scholarship.

Funding: Department of Health, Australian Government: National Health and Medical Research Council (APP1056485)

This is the author manuscript accepted for publication and has undergone full peer review but has not been through the copyediting, typesetting, pagination and proofreading process, which may lead to differences between this version and the Version of Record. Please cite this article as doi: $10.1111 /$ ene.13232

This article is protected by copyright. All rights reserved 


\section{Abstract}

Background: Considered to be benign conditions, the common genetic generalised epilepsy syndromes are now known to be frequently accompanied by cognitive dysfunction. However unresolved issues impede clinical management of this common comorbidity, including which cognitive abilities are most affected, whether there are differences between syndromes, and how seizure-type and mood symptoms affect cognitive dysfunction. We provide a detailed description of cognitive ability and evaluate factors contributing to cognitive dysfunction.

Methods: 76 adults with GGE were assessed with the Woodcock Johnson III Tests of Cognitive Abilities.

Results: Scores on tests of overall cognitive ability, acquired knowledge, long-term retrieval and speed of information processing were significantly below the normative mean. Longterm retrieval was a pronounced weakness with a large reduction in scores $(\mathrm{d}=0.84)$. Neither GGE syndrome, seizure type nor the presence of recent psychopathology symptoms was significantly associated cognitive function.

Conclusions: This study confirms previous meta-analytic findings with a prospective study, offers new insights regarding the cognitive comorbidity of these common epilepsy syndromes and reinforces the need for cognitive interventions in people with GGE.

\section{INTRODUCTION}

Cognitive dysfunction has been consistently reported in people with genetic generalised epilepsy (GGE), a cluster of epilepsy syndromes representing approximately $20 \%$ of all epilepsies [1-3]. A recent meta-analysis of 26 studies showed moderate to large reductions across all cognitive factors, with the greatest deficits in information processing speed, acquired knowledge and fluid intelligence, and working memory [4]. Questions remain regarding the nature of cognitive dysfunction in this cluster of epilepsy syndromes, making it difficult to establish the best method of prevention and treatment of this common comorbidity. It remains unclear whether patterns of cognitive impairment are similar across the main syndromes: childhood absence epilepsy (CAE), juvenile absence epilepsy (JAE), juvenile myoclonic epilepsy (JME) and GGE with generalised tonic-clonic seizures (GTCS) only (GGE-GTCSO).

The lack of objective evidence for neuropsychological differences between these syndromes may be attributable to sampling bias in previous studies, since the majority have focused on 
JME [4]. Studies that have included participants with a range of GGE syndromes rarely conducted comparisons between groups. One familial study of GGE compared CAE and JME, showing few statistically significant differences in cognitive function, limited to some tests of variability in response reaction time and attentional flexibility, both lower in JME [5]. Cognitive functioning in adults with GGE is also under-studied. The estimated nature and extent of dysfunction varies on the basis of sampling and methodological characteristics of individual studies. For example, few studies conducted comprehensive cognitive assessment across all areas of function due to the testing of specific hypotheses regarding executive functioning deficits - a practice which may have led to under-estimation of the extent of cognitive dysfunction $[2,4]$.

The cause of cognitive dysfunction in epilepsy syndromes broadly and GGE more specifically is considered multifactorial and remains uncertain [2]. Possible causes include neurobiological factors such as duration of disease, acute and cumulative effects of seizures, anti-epileptic drug (AED) side-effects, epileptiform discharges and psychopathology comorbidities [6-8]. Ten-year follow-up of people with intractable epilepsy of diverse types has shown frequency of generalised tonic-clonic seizures to be the strongest predictor of cognitive decline [9], although changes in AED use during the follow-up period confound this conclusion [10]. A related finding is the evidence elevated rates of psychopathology in GGE [11-13]. However, there are no studies to our knowledge that have evaluated the extent to which cognitive dysfunction and psychopathology co-occur. With improved understanding, clinical management of psychological dysfunction, cognitive and psychopathological, and effects on quality of life could be addressed.

This study aimed to provide a detailed characterisation of cognitive function in GGE. Comprehensive measurement of cognitive ability factors and psychosocial functioning in a larger, prospective sample of adults with GGE will enable assessment of areas of relative strength and weakness and comparison of cognitive ability between GGE subtypes. Relationships between syndrome, seizure variables, psychopathology and cognition were also measured. 


\section{METHODS}

\section{Participants and procedure}

Patients were recruited prospectively as part of a larger study, through epilepsy specialist clinics at two tertiary hospitals in Melbourne, Australia and a rural clinic [14]. During the period 2011-2015, 120 people with GGE were recruited into the larger prognostic study, 76 of whom underwent cognitive assessment as part of the neuropsychology component of the study (see Online Figure 1). The remaining 44 of those in the larger study either declined to participate in cognitive assessment or were not able to be seen by an investigator at the time of their electroencephalogram (EEG) appointment. There were no significant or clinically meaningful differences between those people who took part in the current study and those from the larger study with respect to demographic or epilepsy characteristics (Online Table I).

We established the diagnosis of GGE according to International League Against Epilepsy (ILAE) criteria, namely, the combination of consistent clinical features and a positive EEG showing generalized epileptiform discharges on at least one occasion [15, 16]. All patients had EEG and brain magnetic resonance imaging (MRI) performed as per routine practice of the epileptologist.

GGE patients were classified into the following categories: childhood absence, juvenile absence, juvenile myoclonic, and generalized epilepsy with generalized tonic-clonic seizures only [15, 16]. Medical records including EEG and neuroimaging were reviewed independently by two epilepsy specialists with any discordance on diagnosis resolved by consensus based on ILAE criteria. Exclusion criteria were: potentially epileptogenic structural abnormalities (such as hippocampal sclerosis), coexistent focal and generalized epilepsies, secondary bilateral synchrony and single seizure with generalized epileptiform abnormalities on EEG.

This research was approved by the Human Ethics Research Committees of participating sites. Participants provided written informed consent as per the Declaration of Helsinki. Investigators collecting cognitive data were blinded to diagnostic and other epilepsy information. 


\section{Cognitive Assessment}

The Woodcock Johnson III Tests of Cognitive Abilities (WJ-III) were used to measure cognitive functioning. These tests were developed on the basis of the comprehensive CattellHorn-Carroll (CHC) model [17]. The CHC model has a demonstrated factor structure that explains cognitive factors underlying the majority of validated cognitive tests, including tests of executive functioning $[18,19]$. Subtests of the WJ-III are combined to form a brief measure of overall intellectual ability (an estimate of IQ), and broad CHC factors: acquired knowledge, long-term retrieval, fluid reasoning, processing speed and short-term memory as described in Table 1 below. Long-term retrieval is synonymous with anterograde memory or new learning. Short-term memory is also known as working memory. Participant test scores were compared to Australian age and demographically adjusted normative data [20]

\section{[Insert Table 1]}

\section{Measurement of Psychopathology Symptoms}

Following cognitive assessment, participants were invited to complete a self-report psychopathology symptom questionnaire, the Adult Self-Report form of the Achenbach System of Empirically Based Assessment [21]. The questionnaire yields six DSM-Oriented Subscales, matched to the Diagnostic and Statistical Manual of Mental Disorders IV-TR. The Achenbach Adult Self-Report form defines T scores 65-69 to correspond to 'borderlineclinical' range, and scores over 69 as 'clinical' range symptoms. This corresponds to $93^{\text {rd }}$ and $97^{\text {th }}$ percentile respectively, thus $7 \%$ of the normative reference group would be expected to endorse borderline-clinical or clinical levels of distress in each DSM-Oriented Subscale [21].

\section{Statistical Analysis}

Distribution plots and one-sample t-tests with Bonferroni corrections for multiple comparisons were used to examine the distributional properties of standard scores on each $\mathrm{CHC}$ factor. To test the hypothesis of specific areas of cognitive strength and deficit in GGE, one-way within-subjects analysis of variance (ANOVA) analysis were employed to test for differences between GGE subgroups on each $\mathrm{CHC}$ factor, with follow-up pairwise t-tests if the omnibus test was significant [22]. 
Multivariate ANOVAs (MANOVAs) were used to test differences between GGE syndromes and seizure types (absence and GTC seizures) on each cognitive factor. Univariate ANOVAs followed when appropriate.

A series of MANOVAs were used to compare scores on all $\mathrm{CHC}$ factors and a global ability index for patients with elevated (borderline clinical or clinical level) symptom scores on any of the DSM-Oriented Subscales, with those who rate within the normal range on these subscales. Pairwise analyses followed when appropriate.

\section{RESULTS}

A total of 76 adolescents and adults with GGE and their subsyndromes (CAE, JAE, JME, GGE-GTCS) completed the cognitive assessment (see Table 2). Two-thirds of the total sample (50 of 76 patients) completed the psychopathology symptom questionnaire. The remaining 26 did not return their questionnaire. The relative frequency of 'borderline-clinical' and 'clinical' level symptom endorsement in each of the six DSM-Oriented Subscales is shown in Table 3.

With respect to education level, the majority of the sample had completed a university degree $(n=31)$ or some vocational training $(n=10)$, a smaller proportion completing only secondary $(n=30)$ or elementary school $(n=2)$. Most were employed $(n=35)$ or studying full-time $(n=23)$, the remainder were unemployed $(n=11)$ or did not disclose employment status $(n=7)$.

[Insert Table 2, Table 3]

Figure 1 and Table 4 show the sample mean of each cognitive ability lies below the normative population value in the relevant age-group, except for short-term memory. Onesample t-tests conducted on each cognitive factor using Bonferroni-adjusted significance value of $0.0083(0.05 / 6)$ revealed significantly lower scores in the GGE group on overall cognitive ability, acquired knowledge, and speed of information processing (small to medium effects), and long-term retrieval (large effect) compared to the norms.

[Insert Figure 1, Table 4]

One-way within-subjects ANOVA comparisons between CHC factor scores in the entire GGE sample showed significant differences between patient scores on the five cognitive factors and the global ability index, $F(5,374)=18.22, p<0.001, \eta^{2}=0.19$ (see Table 5). Bonferroni- 
corrected pairwise comparisons revealed significant, small to medium sized differences between mean scores on a few of the $\mathrm{CHC}$ factor subscales. These within-subject comparisons support the inference from Table 4 that long-term retrieval function is significantly lower than other abilities except speed of information processing, from which it is not significantly different.

\section{[Insert Table 5]}

A series of MANOVAs with clinical covariates as independent variables and the five cognitive functioning factors and global ability index as dependent variables revealed no significant effect of any of the following clinical covariates: GGE syndrome type $(F(4$, $70)=1.16, p=0.29, \eta 2=0.09)$, history of absence seizures $F(1,66)=0.30, p=0.94, \eta 2=0.028)$, or history of GTCS seizures $(F(1,70)=1.24, p=0.30, \eta 2=0.10)$. Online Table II shows cognitive functioning scores separately for these clinical subgroups.

A series of MANOVAs with presence or absence of the six types of psychopathology symptoms as independent variables revealed no statistically significant impact of psychopathology symptoms on cognitive function. Specifically, the models were tested with depressive, anxious, somatic, avoidant, attention deficit, anti-social and total symptoms (see Online Tables III-VIII). The same pattern of null results was obtained when the psychopathology independent variables were reported as continuous.

\section{DISCUSSION}

This study describes cognitive function in adults with GGE. There were three key findings. Firstly, there was a pervasive reduction in cognitive function in people with GGE compared to the local demographically-corrected norms. Performance on tests of overall cognitive ability, and the constituent scores of acquired knowledge, speed of information processing, and long-term retrieval fell significantly below the normative sample by .42 to 0.84 standard deviation units (6 to 13 intelligence index points). Long-term retrieval was a relative weakness compared to other cognitive functions. Fluid intelligence and short-term memory were not significantly different from normative standards. Secondly, we did not find any evidence for the effect of GGE syndrome, history of absence seizures or history of GTCS seizures on cognitive scores. Nor was there evidence of poorer cognitive functioning in subgroups experiencing recent mood symptoms in any of the six DSM-Oriented Subscales. 
Reductions in cognitive functioning in the current prospective cohort mirrored those reported from a recent meta-analysis in both size and direction [4]. One exception relates to the significantly greater deficit in anterograde memory reported by participants in the current study, a common subjective symptom [23], but contrasting with the meta-analytic findings that anterograde memory was relatively spared [4].

In the current sample, smaller, significant reductions in were observed for overall cognitive ability, acquired knowledge and speed of information processing. No significant reduction was observed for short-term memory or fluid intelligence. Short-term memory overlaps with measures of attention, and the lack of significant reduction in our sample with GGE contrasts with findings of attention deficits in children with CAE [2] [24]. There are several possible explanations for this null finding. We may not have detected a difference in short-term memory function because of the relatively small proportion of people with CAE in our adult cohort. Alternatively, there may be differences in the tests used to measure short-term memory function across studies [25] [26]. While attentional deficits are commonly reported in children with CAE, it is possible that attention problems may resolve in adulthood, although this requires further investigation.

The observed reductions in speed of processing, long-term retrieval and acquired knowledge may be related. For example, a possible mechanistic pathway could include a reduction in speed of information processing and memory difficulty which together result in a difficulty retaining learned information, reflected in the reduced acquired knowledge [27, 28]. This combination of deficits has the potential for cumulative and far-reaching educational and vocational disadvantage [29].

A novel aspect of this study involved examination of effects of GGE syndrome and seizure type on cognitive ability. We found no evidence for differences in cognitive ability on the basis of GGE syndrome. This replicates previous null findings of differences between CAE and JME, and across other GGE syndromes $[4,5]$. However the present study may have been underpowered to detect the observed medium sized effect ( 0.7 statistical power), suggesting that future studies should aim for recruitment of at least 25 per GGE syndrome group.

We found no relationship between seizure types and cognitive function. Mean differences in cognitive functioning scores between groups with and without a history of absence seizures were trivial and unlikely to be of clinical significance regardless of sample size. The 
relationship between non-convulsive seizures such as absence, and history of GTCS and cognition, therefore remains a point of debate [9, 30, 31]. It is of course possible that the extent of seizure burden, rather than seizure type contributes to cognitive dysfunction.

The lack of association between mood disturbance and cognitive function is surprising given ample evidence for this relationship in depression and other health conditions [32]. Our findings do not support the concept of shared underlying mechanisms of cognitive and psychosocial comorbidities, or that mood disturbance is a key contributor to cognitive dysfunction in genetic generalised epilepsy. Nonetheless, acute mood states are known to impact retrospective symptom reports significantly due to recall biases, so the two are not entirely independent [33].

This study has a number of methodological strengths: the availability of a relatively large, prospective sample, comprehensive cognitive assessment, and consideration of concurrent mood symptoms on cognition. Limitations include inadequate statistical power to detect significant small to medium sized differences in cognitive function between GGE syndromes and different seizure histories. Although age-matched local Australian norms were used, the accuracy of our estimates of cognitive deficits may have been improved with a large studyrecruited control group. Future research should consider healthy and sibling control groups while retaining representation from all GGE syndromes. The inclusion of a sibling control group may enable consideration of potential underlying genetic factors unrelated to epilepsy.

Assuming a normal distribution, the reduction of overall cognitive functioning of six standard score points in this GGE sample suggests the prevalence of intellectual disability (intelligence test score of less than or equal to 70) of approximately 5.5\% compared to the general population prevalence of approximately $2 \%$. On this basis an estimated additional $12 \%$ of people with GGE would be expected to have 'borderline' range intellectual functioning (intelligence test scores of 70-80), above the population prevalence of approximately $7 \%$. This estimated cumulative prevalence of cognitive ability in the range of 'borderline' or intellectually disability of $17.5 \%$. Our findings replicate previous metaanalytic findings of cognitive dysfunction in GGE in a prospective sample. The results further reinforce the need for screening of cognitive dysfunction in adults with GGE, even after initial diagnosis, and the provision of support for memory and other cognitive difficulties. 


\section{References}

1. Jallon P, Latour P. Epidemiology of idiopathic generalized epilepsies. Epilepsia. 2005;46 Suppl 9:10-4.

2. Hommet C, Sauerwein HC, De Toffol B, Lassonde M. Idiopathic epileptic syndromes and cognition. Neurosci Biobehav Rev. 2006;30(1):85-96.

3. Cutting S, Lauchheimer A, Barr W, Devinsky O. Adult-onset idiopathic generalized epilepsy: clinical and behavioral features. Epilepsia. 2001;42(11):1395-8.

4. Loughman A, Bowden S, D'Souza W. Cognitive functioning in idiopathic generalised epilepsies: A systematic review and meta-analysis. Neurosci Biobehav Rev. 2014;43:20-34.

5. Levav M, Mirsky AF, Herault J, Xiong L, Amir N, Andermann E. Familial association of neuropsychological traits in patients with generalized and partial seizure disorders. Journal of clinical and experimental neuropsychology. 2002;24(3):311-26.

6. Caplan R, Siddarth P, Stahl L, Lanphier E, Vona P, Gurbani S, et al. Childhood absence epilepsy: behavioral, cognitive, and linguistic comorbidities. Epilepsia. 2008;49(11):1838-46.

7. Dlugos D, Shinnar S, Cnaan A, Hu F, Moshé S, Mizrahi E, et al. Pretreatment EEG in childhood absence epilepsy Associations with attention and treatment outcome. Neurology. 2013;81(2):150-6.

8. Bhise VV, Burack GD, Mandelbaum DE. Baseline cognition, behavior, and motor skills in children with new-onset, idiopathic epilepsy. Developmental Medicine \& Child Neurology. 2010;52(1):22-6.

9. Thompson PJ, Duncan JS. Cognitive decline in severe intractable epilepsy. Epilepsia. 2005;46(11):1780-7.

10. Bergen DC. Do Seizures Harm the Brain? Epilepsy Curr. 2006;6(4):117-8. 
11. Loughman A, Bendrups N, D'Souza W. A systematic review of psychiatric and associated comorbidities of idiopathic generalised epilepsies. Neuropsychology Review. 2016; In press.

12. Akanuma N, Hara E, Adachi N, Hara K, Koutroumanidis M. Psychiatric comorbidity in adult patients with idiopathic generalized epilepsy. Epilepsy Behav. 2008;13(1):248-51.

13. Camfield P, Camfield C. Idiopathic generalized epilepsy with generalized tonic-clonic seizures (IGE-GTC): a population-based cohort with $>20$ year follow up for medical and social outcome. Epilepsy \& Behavior: E\&B. 2010;18(1-2):61-3.

14. Seneviratne U, Hepworth G, Cook M, D'Souza W. Atypical EEG abnormalities in genetic generalized epilepsies. Clin Neurophysiol. 2015.

15. ILAE CoCaT. Proposal for revised classification of epilepsies and epileptic syndromes. Commission on Classification and Terminology of the International League Against Epilepsy. Epilepsia. 1989;30(4):389-99.

16. Berg AT, Berkovic SF, Brodie MJ, Buchhalter J, Cross JH, Van Emde Boas W, et al. Revised terminology and concepts for organization of seizures and epilepsies: report of the ILAE Commission on Classification and Terminology, 2005-2009. Epilepsia. 2010;51(4):676-85.

17. McGrew KS, Woodcock RW. Woodcock-Johnson III technical manual: Riverside Pub.; 2001

18. Jewsbury PA, Bowden SC, Strauss ME. Integrating the switching, inhibition, and updating model of executive function with the Cattell-Horn-Carroll model. J Exp Psychol Gen. 2016;145(2):220-45.

19. Jewsbury PA, Bowden SC, Duff K. The Cattell-Horn-Carroll Model of Cognition for Clinical Assessment. J Psychoeduc Assess. 2016:0734282916651360.

20. McGrew KS. The Australian Standardisation of the Woodcock-Johnson III Cognitive and Achievement Battery. Australian Psychological Society Annual Conference; 23 September 2008; Hobart, Tasmania2008. 
21. Achenbach, Rescorla L. Manual for the ASEBA adult forms \& profiles. Burlington, VT: University of Vermont, Research Center for Children, Youth, \& Families. 2003.

22. Tabachnick BG, Fidell LS. Using Multivariate Statistics: Pearson; 2007.

23. Fisher RS, Vickrey BG, Gibson P, Hermann B, Penovich P, Scherer A, et al. The impact of epilepsy from the patient's perspective I. Descriptions and subjective perceptions. Epilepsy Research. 2000;41(1):39-51.

24. Vega C, Vestal M, DeSalvo M, Berman R, Chung M, Blumenfeld H, et al. Differentiation of attention-related problems in childhood absence epilepsy. Epilepsy Behav. 2010;19(1):82-5.

25. Engle R, Tuholski S, Laughlin J, Conway ARA. Working memory, short-term memory, and general fluid intelligence: A latent-variable approach. Journal of experimental psychology General. 1999;128(3):309-31.

26. Conway ARA, Cowan N, Bunting M, Therriault D, Minkoff SRB. A latent variable analysis of working memory capacity, short-term memory capacity, processing speed, and general fluid intelligence. Intelligence. 2002;30(2):163-83.

27. Lichtenberger EO, Kaufman AS. Essentials of WAIS-IV assessment: John Wiley \& Sons; 2009.

28. Sattler JM. Assessment of children: Behavioral and clinical applications: Jerome M Sattler Publisher; 2002.

29. Lezak MD. Neuropsychological assessment: Oxford University Press, USA; 2004.

30. Aminoff MJ. DO nonconvulsive seizures damage the brain?-no. Arch Neurol. 1998;55(1):119-20.

31. Young G, Jordan KG. DO nonconvulsive seizures damage the brain?-yes. Arch Neurol. 1998;55(1):117-9.

32. McDermott L, Ebmeier K. A meta-analysis of depression severity and cognitive function. Journal of affective disorders. 2009;119(1):1-8.

33. Gorin A, Stone A. Recall biases and cognitive errors in retrospective self-reports: A call for momentary assessments. Handbook of health psychology. 2001;23:405-13. 
Table 1. Broad CHC Factors and corresponding Woodcock Johnson III Subtests

\begin{tabular}{|c|c|c|}
\hline Broad CHC Factor & $\begin{array}{l}\text { Narrow Abilities } \\
\text { Measured }\end{array}$ & $\begin{array}{l}\text { Cluster of WJ-III Tests of Cognitive } \\
\text { Abilities }\end{array}$ \\
\hline $\begin{array}{l}\text { Comprehension- } \\
\text { Knowledge (Gc) }\end{array}$ & $\begin{array}{l}\text { Lexical knowledge, } \\
\text { Language development }\end{array}$ & Test 1: Verbal Comprehension \\
\hline . & $\begin{array}{l}\text { General (verbal) } \\
\text { information }\end{array}$ & Test 11: General Information \\
\hline Long-Term F & Associative memory & $\begin{array}{l}\text { Test 2: Visual-Auditory Learning } \\
\text { Test 10: Visual-Auditory Learning } \\
\text { Delayed }\end{array}$ \\
\hline & Ideational fluency & Retrieval Fluency \\
\hline Fluid Reason & $\begin{array}{l}\text { Induction } \\
\text { General sequential } \\
\text { reasoning }\end{array}$ & $\begin{array}{l}\text { Test 5: Concept Formation } \\
\text { Test 15: Analysis Synthesis }\end{array}$ \\
\hline Processing Sp & $\begin{array}{l}\text { Perceptual Speed } \\
\text { Attention and } \\
\text { concentration }\end{array}$ & $\begin{array}{l}\text { Test 6: Visual Matching } \\
\text { Test 20: Pair Cancellation }\end{array}$ \\
\hline $\begin{array}{l}\text { Short-Term Memory } \\
(\mathrm{Gsm})\end{array}$ & Working memory & $\begin{array}{l}\text { Test 7: Numbers Reversed } \\
\text { Test 9: Auditory Working Memory }\end{array}$ \\
\hline & Memory span & Test 17: Memory for Words \\
\hline
\end{tabular}

Table 2. Patient demographics $n=76$

\section{Variables}

Age (years)

Range

$13-58$

Mean (SD)

$29.0(11.2)$

Gender $n(\%)$

$\mathrm{M}$

$\mathrm{F}$

Syndrome $\mathrm{n}(\%)$

CAE

JAE
$26(34 \%)$

$50(66 \%)$

$10(13 \%)$

$21(28 \%)$ 
JME

GTCSO

Other

Current AED n(\%)

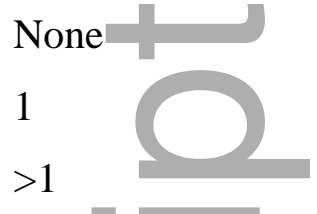

Lamotrigine

Valproate

Levetiracetam

Other AED

History of absence seizures $\left(n=69^{\wedge}\right)$

No

Yes

History of GTCS $\left(n=73^{\wedge}\right)$

No

Yes

Days since last GTCS $\left(n=69^{\wedge}\right)$

Range

IQR

\section{(1)}

Seizure free duration (days) $\left(n=69^{\wedge}\right)$

Range

IQR
$20(26 \%)$

$23(30 \%)$

$2(3 \%)$

$10(13 \%)$

$34(45 \%)$

$32(42 \%)$

27

39

14

17

$35(51 \%)$

$34(49 \%)$

$8(11 \%)$

$65(89 \%)$

3-9855

1340

1-9855

633.5

* AEDs include: Clonazepam, Topiramate, Carbamazepine, Zonisamide, Piracetam, Vimpat NB: some clinical information was unavailable for up to seven patients.

Table 3. Frequency of psychopathology symptoms $n=50$. Figures refer to percentages

Symptom type

Depressive

Anxious

Somatic

Clinical

Borderline-Clinical

Normal 
Avoidant

ADHD

8

14

Antisocial Personality

6

6

94

Total problems

16

10

74

Table 4. One-sample t-test and effect sizes for cognitive functioning in GGE $n=76$

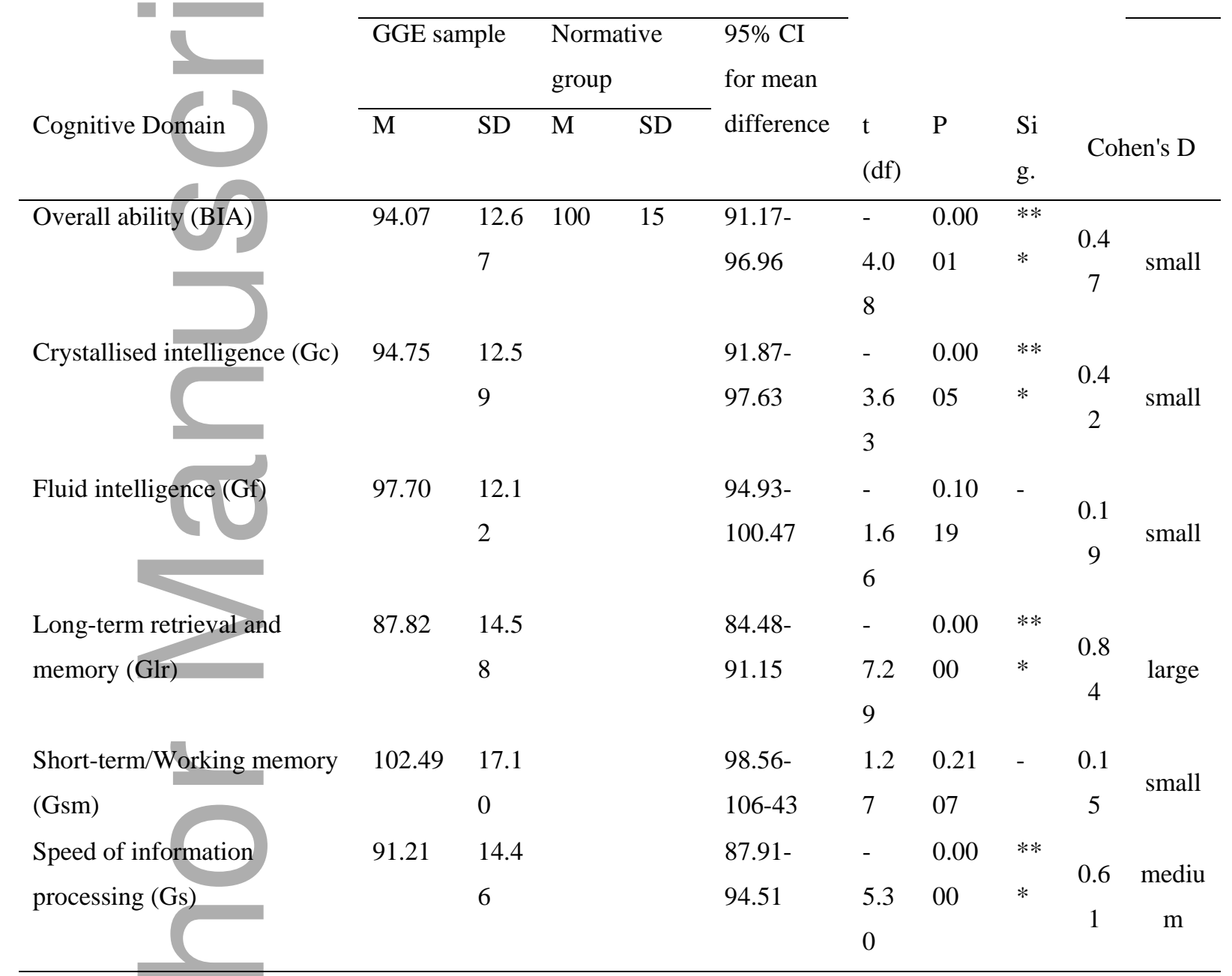

Two-tailed significance - n.s.

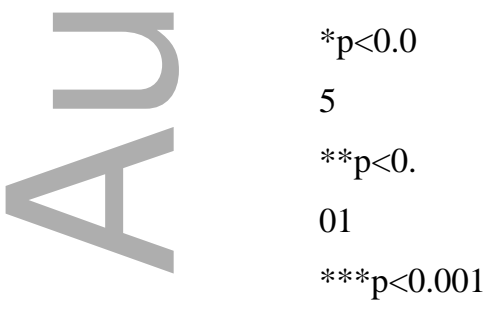

This article is protected by copyright. All rights reserved 
Table 5. Pairwise comparisons between CHC factor scores within the entire GGE sample, with Bonferroni correction. (See Table 4 for CHC factor domain abbreviations).

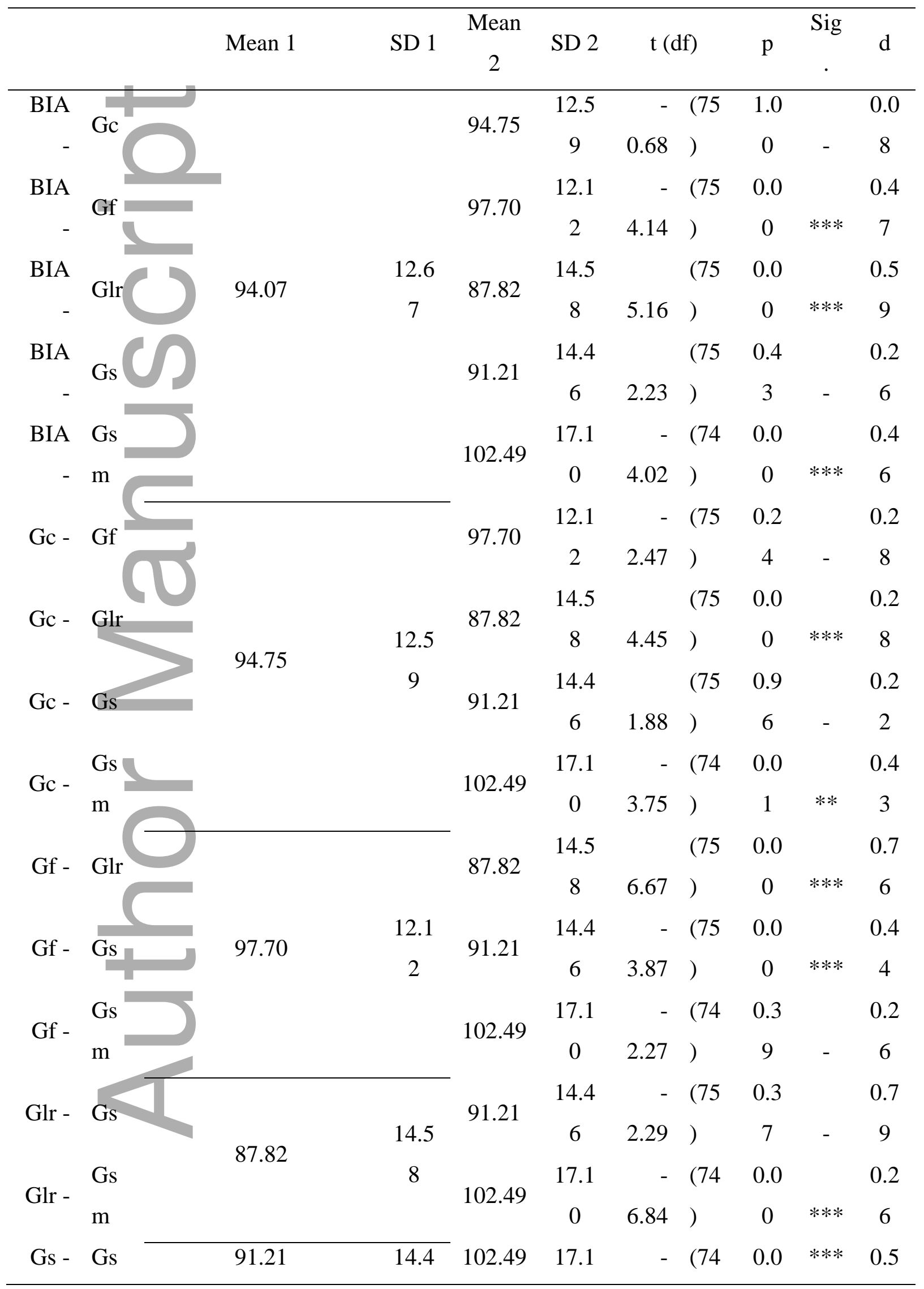




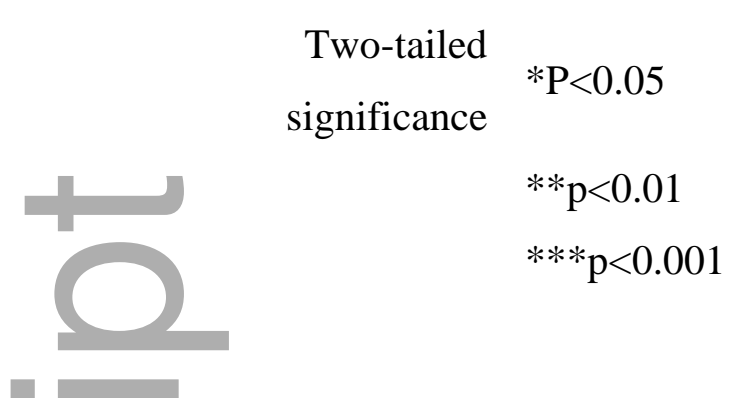

\section{Figure Legend}

Figure 1. Distribution plots of each $\mathrm{CHC}$ factor The normative mean of 100 is indicated by a red vertical line while the mean and distributional properties of scores on each $\mathrm{CHC}$ factor are indicated by the dotted line and shaded colours

\section{Supplementary Material}

Online Figure 1. Flow chart of recruitment and participation

Online Table I. Comparison of demographic and clinical information in sample with and without cognitive assessment.

Online Table II. Cognitive functioning by GGE syndromes and seizure type

Online Table HI-VIII. Cognitive functioning in subgroups with and without borderline clinical/clinical symptoms

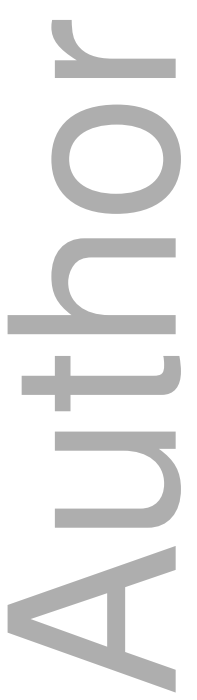



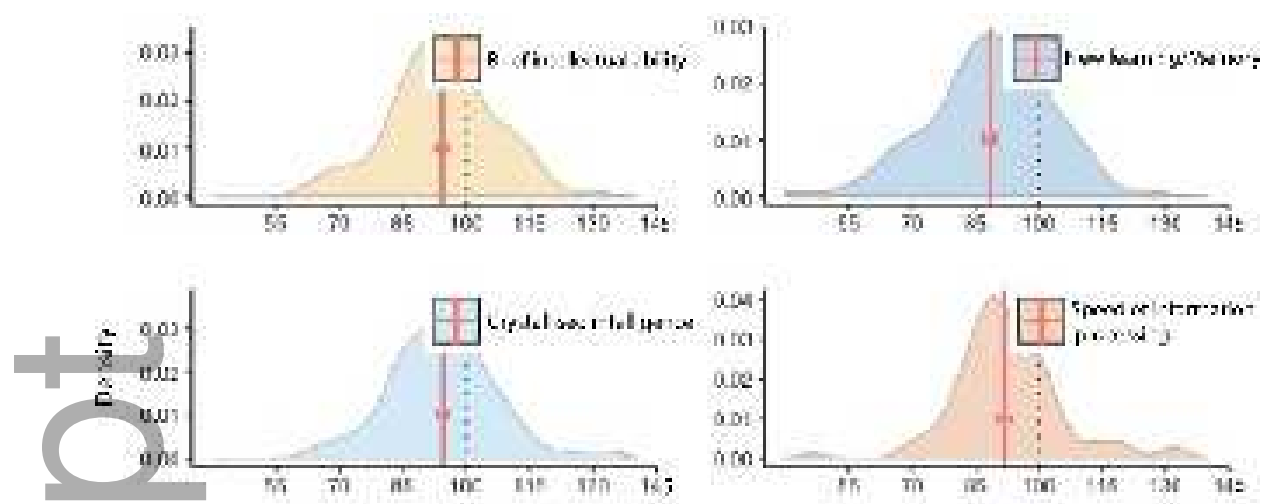

$$
\square
$$
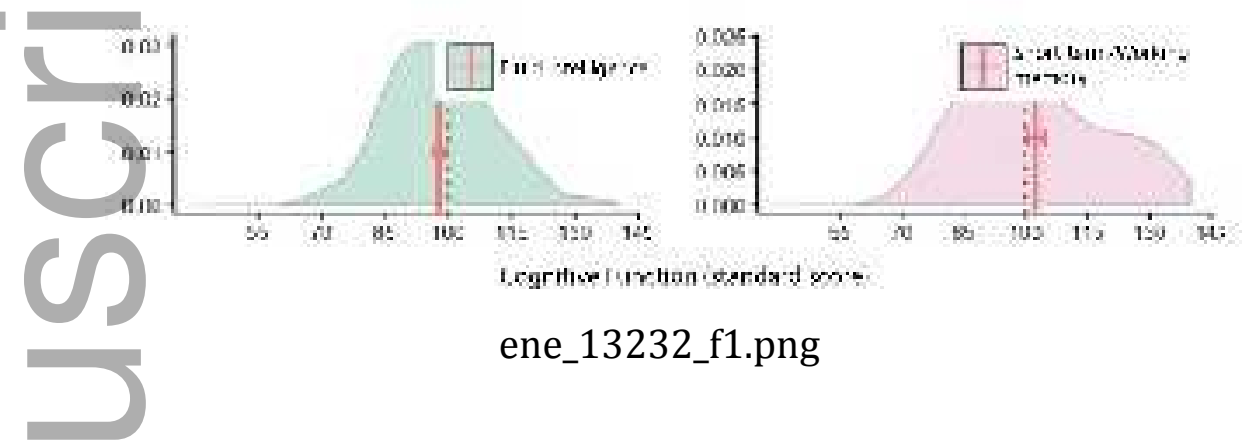

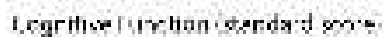

ene_13232_f1.png
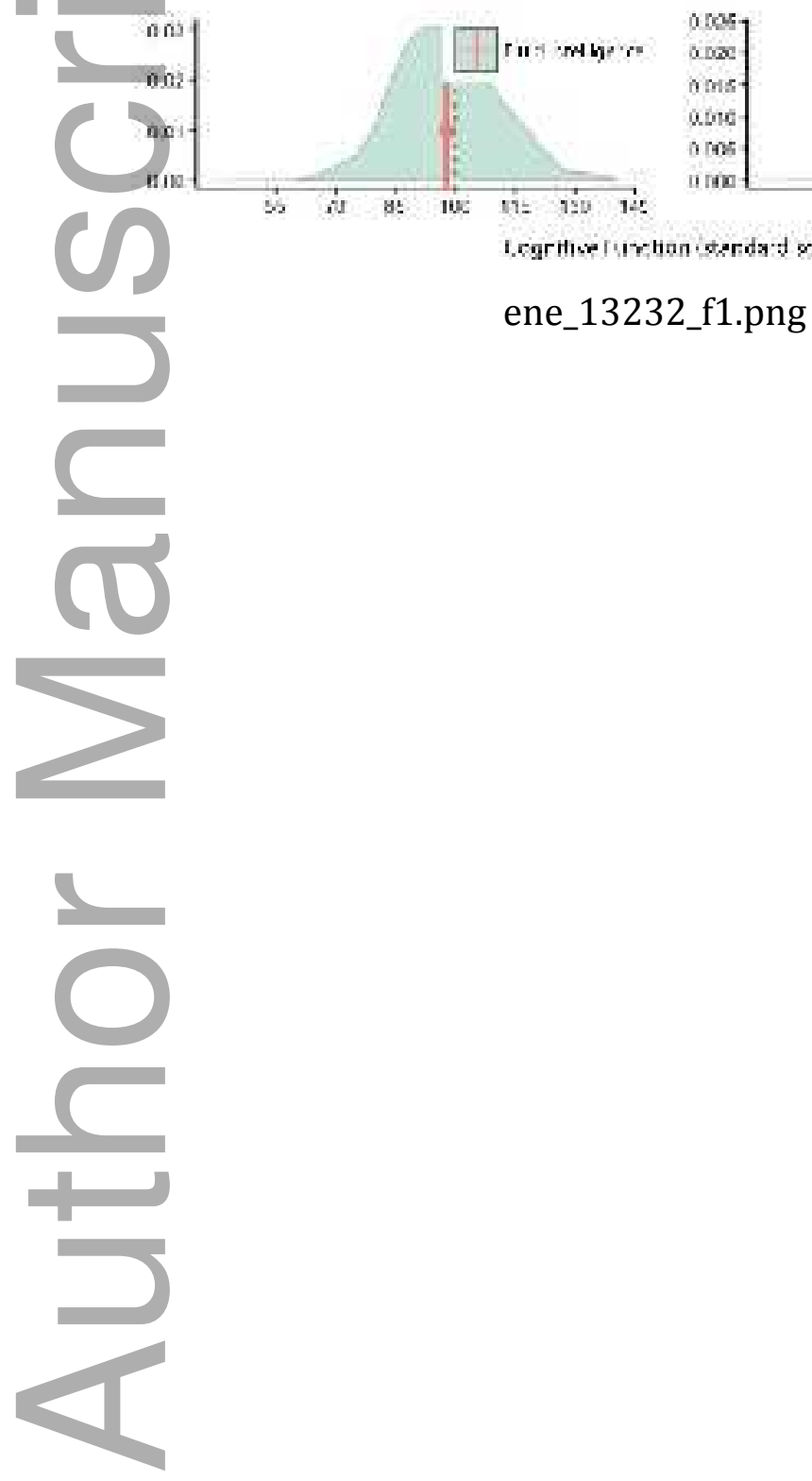


\section{University Library}

\section{- M M N E R VA A gateway to Melbourne's research publications}

Minerva Access is the Institutional Repository of The University of Melbourne

Author/s:

Loughman, A;Bowden, SC;D'Souza, WJ

Title:

A comprehensive assessment of cognitive function in the common genetic generalized epilepsy syndromes

Date:

2017-03-01

Citation:

Loughman, A., Bowden, S. C. \& D'Souza, W. J. (2017). A comprehensive assessment of cognitive function in the common genetic generalized epilepsy syndromes. EUROPEAN JOURNAL OF NEUROLOGY, 24 (3), pp.453-460. https://doi.org/10.1111/ene.13232.

Persistent Link:

http://hdl.handle.net/11343/292262 